\title{
Activities of Daily Living after Surgical Treatment for Osteoporotic Vertebral Fracture with or without Diffuse Idiopathic Skeletal Hyperostosis: A Retrospective Single-Institutional Study
}

\author{
Shinichi Kato, Nobuki Terada, Osamu Niwa \\ Department of Orthopedic Surgery, Restorative Medicine of Neuro-Musculoskeletal System, Fujita Health University School of Medicine, Nagoya, Japan
}

Study Design: This investigation was a retrospective observational study.

Purpose: The aim of this study was to evaluate whether having diffuse idiopathic skeletal hyperostosis (DISH) as a comorbidity affects the patient's ability to perform activities of daily living (ADL) after surgical treatment for osteoporotic vertebral fracture (OVF). Overview of Literature: A few studies have extensively evaluated elderly patients with comorbidities such as DISH and OVFinduced persistent back pain and their ability to perform ADL postoperatively.

Methods: In this study, 63 patients (21 men and 42 women) who underwent surgical treatment for OVF were enrolled. Of these patients, 26 had DISH (D+) and 37 did not have DISH (D-). Patient demographic characteristics and surgical, clinical, and radiological findings were compared between those with and without DISH. The change in their ability to perform ADL after surgery was also evaluated.

Results: Age, number of comorbidities, and 1-year mortality rate were significantly higher in the $\mathrm{D}+$ group $(p<0.05)$. Postoperative Visual Analog Scale (VAS) scores were significantly higher in patients with impaired ( $n=6, p=0.04$ ) abilities to perform ADL, and improvements in VAS scores were significantly higher in patients with unchanged abilities to perform $\operatorname{ADL}(n=54, p=0.03)$ after surgery. The average postoperative VAS scores were 2.2 for the D+ group and 2.3 for the D- group, which were not significantly different. Conclusions: The frequency of OVF with DISH was higher in elderly men with multiple comorbidities and contributed to a higher 1 -year mortality rate than those in patients without DISH. However, preoperative and postoperative VAS scores and improvements in VAS scores were similar between those with and without DISH. Postoperative impaired ability to perform ADL was associated with old age, high postoperative VAS scores, and little improvements in VAS scores, which were limitedly influenced by DISH. Surgical treatment of OVF combined with DISH is effective and appropriate for elderly patients.

Keywords: Diffuse idiopathic skeletal hyperostosis; Osteoporotic fractures; Comorbidity; Visual Analog Scale; Activities of daily living

\section{Introduction}

Osteoporotic vertebral fracture (OVF) is the most com- mon osteoporotic fracture and a possible risk factor for shortening a healthy life expectancy. Although several patients with OVF achieve remission after conservative

Received Dec 4, 2019; Revised Jan 24, 2020; Accepted Jan 31, 2020

Corresponding author: Shinichi Kato

Department of Orthopedic Surgery, Restorative Medicine of Neuro-Musculoskeletal System, Fujita Health University School of Medicine, 3-6-10 Otobashi, Nakagawaku, Nagoya, Aichi, 454-8509, Japan

Tel: +81-52-323-5650, Fax: +81-52-332-8634, E-mail: shkato@fujita-hu.ac.jp 
treatment, some require surgery due to an impairment in their ability to perform activities of daily living (ADL) as a consequence of residual pain attributable to delayed union of the fracture. As the majority of these patients are elderly and have low physical function, it is necessary to carefully evaluate their comorbidities and complications.

Diffuse idiopathic skeletal hyperostosis (DISH), which involves reduced range of motion of an intervertebral disc caused due to ankylosing spondylitis, is a particularly common comorbidity. Although vertebral fractures complicated by DISH are associated with poor survival rates, there is a lack of studies addressing a detailed examination of the effects of DISH on the surgical treatment of OVF or the relationship between DISH and ADL. Herein, we hypothesized that having DISH as a comorbidity would have a negative impact on the patient's ability to perform ADL after the surgical treatment for OVF. Thus, the objectives of this study were to explore the clinical features of OVF and the effects of DISH on surgical outcomes and to identify the factors that affect the ability to perform ADL postoperatively.

\section{Materials and Methods}

\section{Patient population}

This research was a retrospective observational study conducted in compliance with the Declaration of Helsinki. The study's protocol was reviewed and approved by the Institutional Review Board for Clinical Research Ethics at Fujita Health University (approval no., HM 18-120) and written informed consent was obtained.

A total of 63 patients (21 men and 42 women) who had undergone surgical treatment for OVF associated with DISH (D+, 26 patients) or not associated with DISH (D-, 37 patients) between April 2010 and April 2018 were enrolled in this study. The average age of these patients was 76.6 years (range, 53-93 years), and the average observation period was 27.9 months (range, 4-77 months). The levels of the affected vertebrae were the thoracolumbar transitional vertebrae in 40 patients and the middle and lower lumbar vertebrae in 23 patients. The ability to perform ADL was divided into the following four categories: independent lifestyle ( $\operatorname{rank} \mathrm{J})$, requires help ( $\operatorname{rank} \mathrm{A}$ ), semi-bedridden (rank B), and bedridden (rank C). The ability to perform ADL before injury and at 6 months postoperatively was compared and evaluated in terms of maintained or impaired ability to perform ADL.

\section{Inclusion and exclusion criteria}

The inclusion criteria were patients with OVF-induced neurological deficits and persistent back pain causing an impaired ability to perform ADL. The diagnosis of DISH was defined as continuous ossification of more than four spinal vertebrae according to the criteria described by Resnick et al. [1]. The exclusion criteria were patients with ossification of the sacroiliac joint, history of spine surgery, and pathological fracture of the metastatic vertebra.

\section{Surgical technique}

The surgical procedures consisted of posterior spinal fusion (PSF) and spinal implants for all patients. Posterior approach decompression was performed for those with OVF when there were accompanying neurological deficits involving the spinal canal fragments. As a general principle in surgical procedures, for patients in whom the thoracolumbar transitional vertebrae were involved, PSF was performed two levels above and below the affected vertebra without corrective force. Vertebroplasty (VP) was also performed when the affected vertebra contained a cleft. For patients in whom the middle and lower lumbar vertebrae were involved, PSF was performed at one level above and below the affected vertebra when anterior column reconstruction was performed. Posterior lumbar interbody fusion (PLIF) was also performed when an intervertebral cleft or instability was detected around the affected vertebra. Anterior fixation with vertebral replacement was performed for patients with massive vertebral collapse (Fig. 1). The fixation range was extended as appropriate when cases were complicated by previous vertebral fractures in the upper and lower PSF ends.

\section{Patient demographic characteristics and surgical, clinical, and radiological examinations}

The demographic characteristics and the examination results of all patients were compared between those with DISH and those without DISH. Demographic characteristics included age, sex, follow-up period, period from injury to surgery (waiting period), changes in neurological deficits, and comorbidities. Surgical characteristics included the surgical procedure, operative time, surgical 

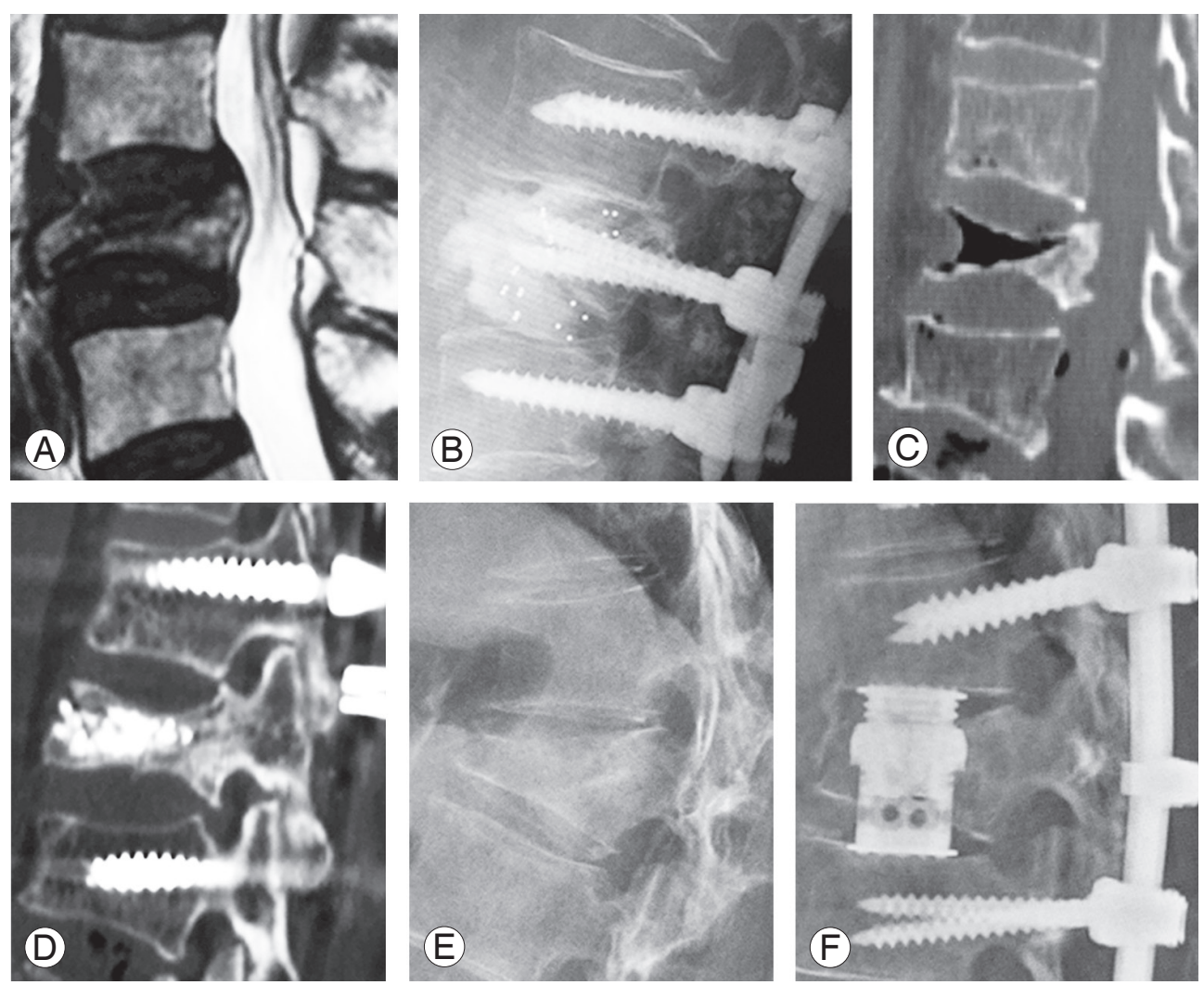

Fig. 1. Anterior column reconstruction. (A) OVF at $L 4$ with an intervertebral instability observed on the magnetic resonance imaging T2-weighted sagittal plane. (B) Posterior fixation from $L 3$ to $L 5$ and posterior lumbar interbody fusion. (C) OVF at Th12 with a cleft observed on the computed tomography sagittal plane. (D) Posterior fixation from Th10 to L2 and vertebroplasty. (E) OVF at L1 with a massive vertebral collapse observed on the X-ray lateral view. (F) Posterior fixation from Th11 to L3 and anterior fixation with vertebral replacement. OVF, osteoporotic vertebral fracture.

blood loss, fusion level, perioperative complications, and revision surgery. The indication of revision surgery for implant failure was implant dislocation accompanied by clinical symptoms; this occurred immediately after surgical treatment. The clinical characteristics included changes in the Visual Analog Scale (VAS) score, ability to perform $\mathrm{ADL}$, and 1-year mortality. In addition, the radiological characteristics included previous vertebral fractures, three-column injury, middle column injury, corrective angle, corrective loss, additional vertebral fracture, and OVF fusion.

Additional vertebral fractures, OVF fusion, and ability to perform ADL were diagnosed in 60 patients who were followed up for more than 6 months postoperatively. All the abovementioned clinical, surgical, and radiological outcomes of patients with unchanged and impaired abilities to perform ADL were compared.

\section{Statistical analysis}

The data of patients with and without DISH and those of patients with unchanged and impaired abilities to perform ADL were statistically analyzed and compared. The Mann-Whitney test was used to analyze the following continuous variables: age, follow-up period, waiting period, comorbidity, operative time, surgical blood loss, fusion level, VAS score, corrective angle, and corrective loss. The classification variables, including neurological disorders, surgical procedure, perioperative complications, revision surgery, ability to perform ADL, 1-year mortality, previous vertebral fracture, three-column injury, middle column injury, additional vertebral fracture, and OVF fusion, were analyzed using the chi-square or Fisher's exact test. The JMP ver. 12.0.1 software (SAS Institute Inc., Cary, NC, USA) was used for statistical analyses, and the level of statistical significance was set at $p<0.05$. 


\section{Results}

\section{Demographic characteristics of patients with and without diffuse idiopathic skeletal hyperostosis}

The average age of patients in the $\mathrm{D}+$ group ( 80.5 years) was significantly higher $(p=0.005)$ than that of the $\mathrm{D}-$ group (73.9 years). Men comprised a significantly greater proportion in the $\mathrm{D}+$ group $(50 \%)(p=0.01)$ than in the D- group (21.6\%). Among the study patients, 33 (D+, 15 patients; $\mathrm{D}-, 18$ patients) had preoperative neurological disorders, 31 exhibited improvement after surgery, and the other two patients ( $\mathrm{D}+$, one patient; $\mathrm{D}-$, one patient) showed no change. These differences were not statistically significant. Patients in the $\mathrm{D}+$ group had a significantly greater number of comorbidities (2.6 diseases, $p=0.02$ ) than those in the $\mathrm{D}$ - group (1.6 diseases) (Table 1).

\section{Surgical outcomes of patients with and without dif- fuse idiopathic skeletal hyperostosis}

A total of 10 patients in the $\mathrm{D}+$ group and 18 patients in

Table 1. Patient demographics in comparison between with DISH and without DISH

\begin{tabular}{lccc} 
Characteristic & $\begin{array}{c}\text { DISH (+) } \\
(\mathrm{n}=26)\end{array}$ & $\begin{array}{c}\text { DISH (-) } \\
(\mathrm{n}=37)\end{array}$ & $p$-value \\
\hline Age (yr) & $80.5 \pm 5.8$ & $73.9 \pm 10.4$ & $0.005^{*}$ \\
\hline Sex & 13 & 8 & \\
\hline \multicolumn{1}{|c|}{ Male } & 13 & 29 & \\
\hline Female & $26.8 \pm 21.1$ & $29.4 \pm 19.7$ & 0.62 \\
\hline Follow-up period (mo) & $2.9 \pm 2.6$ & $3.6 \pm 3.3$ & 0.34 \\
\hline Waiting period (mo) & 15 & 18 & 0.38 \\
\hline Preoperative neurological disorders & & & \\
\hline Frankel score & 1 & - & \\
\hline B & 8 & 12 & \\
\hline C & 6 & 6 & \\
\hline D & 4 & 5 & 0.63 \\
\hline Postoperative neurological disorders & & & \\
\hline Frankel score & 2 & 1 & \\
\hline C & 2 & 4 & \\
\hline D & $2.6 \pm 1.7$ & $1.6 \pm 1.4$ & $0.02^{*}$ \\
\hline Comorbidity & & & \\
\hline
\end{tabular}

Values are presented as mean \pm standard deviation or number. DISH, diffuse idiopathic skeletal hyperostosis.

$p<0.05$; statistically significant. the D- group underwent PSF. Both PSF and VP were conducted for nine patients in the $\mathrm{D}+$ group and six patients in the D- group. Furthermore, five patients in the D+ group and seven patients in the D- group underwent PSF and PLIF. Anterior-posterior fusion was performed for two patients in the $\mathrm{D}+$ group and six patients in the $\mathrm{D}-$ group. The average operative time was 209 minutes in the $\mathrm{D}+$ group and 244 minutes in the $\mathrm{D}-$ group, and the average blood loss volume was $402 \mathrm{~mL}$ in the $\mathrm{D}+$ group and $408 \mathrm{~mL}$ in the $\mathrm{D}$ - group. The average fusion level was 4.5 in the $\mathrm{D}+$ group and 4.4 in the $\mathrm{D}$ - group. Perioperative complications occurred in 14 patients in the D+ group and 12 patients in the $\mathrm{D}$ - group. Revision surgery was required for six patients in the $\mathrm{D}+$ group and three patients in the $\mathrm{D}$ - group. All these differences were not statistically significant (Table 2).

\section{Clinical outcomes of patients with and without dif- fuse idiopathic skeletal hyperostosis}

The average preoperative VAS scores were 7.0 for patients

Table 2. Surgical outcomes of patients in comparison between with DISH and without DISH

$\begin{array}{ccc}\text { Variable } & \text { DISH }(+) & \text { DISH }(-) \\ (n=26) & (n=37) & p \text {-value }\end{array}$

Surgical procedure

0.46

\begin{tabular}{lccc}
\hline PSF & 10 & 18 & \\
\hline PSF+VP & 9 & 6 & \\
\hline PSF+PLIF & 5 & 7 & \\
\hline APF & 2 & 6 & \\
\hline Operative time (min) & $209 \pm 85$ & $244 \pm 100$ & 0.15 \\
\hline Surgical blood loss (mL) & $402 \pm 64$ & $408 \pm 54$ & 0.94 \\
\hline Fusion level & $4.5 \pm 0.4$ & $4.4 \pm 0.3$ & 0.73 \\
\hline Perioperative complication & 14 & 12 & 0.07 \\
\hline Delayed wound healing & 5 & 4 & \\
\hline Implant failure & 4 & 6 & \\
\hline Wound infection & 3 & 1 & \\
\hline Revision surgery & 6 & 3 & 0.09 \\
\hline Delayed wound healing & 0 & 1 & \\
\hline Implant failure & 4 & 1 & \\
\hline Wound infection & 2 & 1 & \\
\hline
\end{tabular}

Values are presented as number or mean \pm standard deviation.

DISH, diffuse idiopathic skeletal hyperostosis; PSF, posterior spinal fusion; VP, vertebroplasty; PLIF, posterior lumbar interbody fusion; APF, anterior and posterior fixation. 
in the $\mathrm{D}+$ group and 7.6 for those in the $\mathrm{D}$ - group. Postoperatively, the average VAS scores were 2.2 for patients the $\mathrm{D}+$ group and 2.3 for those in the $\mathrm{D}$ - group. The average improvement rates in the VAS score were $67.1 \%$ for the $\mathrm{D}+$ group and $67.7 \%$ for the $\mathrm{D}$ - group. Regarding the ability to perform ADL, it was unchanged in 22 patients in the $\mathrm{D}+$ group and 32 patients in the $\mathrm{D}$ - group, whereas it was impaired in two patients in the $\mathrm{D}+$ group and four patients in the D- group. These differences were also not statistically significant. However, the 1-year mortality rate was significantly higher $(p=0.02)$ in the $\mathrm{D}+$ group (three patients) than in the $\mathrm{D}$ - group (0 patients) (Table 3 ).

\section{Radiological outcomes of patients with and without diffuse idiopathic skeletal hyperostosis}

Previous vertebral fractures were detected in 11 patients in the $\mathrm{D}+$ group and 16 patients in the $\mathrm{D}$ - group. Threecolumn injuries were found in 14 patients in the $\mathrm{D}+$ group and 15 patients in the $\mathrm{D}$ - group, and middle column injuries were found in 16 patients in the D+ group and 28 patients in the $\mathrm{D}$ - group. The average corrective angle was $17.2^{\circ}$ in the $\mathrm{D}+$ group and $14.3^{\circ}$ in the $\mathrm{D}$ - group. These differences were not statistically significant. However, the average corrective loss was significantly lower $(p=0.02)$ in the $\mathrm{D}+$ group $\left(6.4^{\circ}\right)$ than in the $\mathrm{D}$ - group $\left(11.1^{\circ}\right)$.

Of the 60 patients who were followed up for 1 year postoperatively, two patients in the $\mathrm{D}+$ group and eight patients in the D- group experienced additional vertebral fractures, but the difference was not significant. OVF fusion was observed at a significantly higher frequency

Table 3. Clinical outcomes of patients in comparison between with DISH and without DISH

\begin{tabular}{lccc|} 
Variable & $\begin{array}{c}\text { DISH (+) } \\
(\mathrm{n}=26)\end{array}$ & $\begin{array}{c}\text { DISH (-) } \\
(\mathrm{n}=37)\end{array}$ & $p$-value \\
\hline Preoperative VAS (cm) & $7.0 \pm 1.6$ & $7.6 \pm 1.8$ & 0.21 \\
\hline Postoperative VAS (cm) & $2.2 \pm 1.2$ & $2.3 \pm 1.1$ & 0.6 \\
\hline $\begin{array}{l}\text { Improvement rate of VAS (\%) } \\
\text { Activity of daily living ability }\end{array}$ & $67.1 \pm 20.9$ & $67.7 \pm 21.4$ & 0.86 \\
\hline Unchanged & 22 & 32 & 0.88 \\
\hline Impaired & $2^{\text {a) }}$ & $4^{\text {b) }}$ & \\
\hline 1-Year mortality & 3 & 0 & $0.02^{*}$ \\
\hline
\end{tabular}

Values are presented as mean \pm standard deviation or number. DISH, diffuse idiopathic skeletal hyperostosis; VAS, Visual Analog Scale.

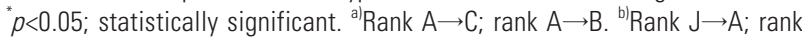
$\mathrm{A} \rightarrow \mathrm{B}$; rank $\mathrm{A} \rightarrow \mathrm{B}$; rank $\mathrm{A} \rightarrow \mathrm{B}$.
( $p=0.004)$ in the $\mathrm{D}+$ group (21/26 patients) than in the $\mathrm{D}-$ group (20/37 patients) (Table 4$)$.

\section{Demographic characteristics and surgical, clini- cal, and radiological outcomes of patients with un- changed and impaired abilities to perform activities of daily living}

There were 54 patients in the group with unchanged ability to perform $\mathrm{ADL}$ and six patients in the group with impaired ability to perform ADL. The average age (75.7 years) of patients with unchanged ability was significantly lower $(p=0.01)$ than that of patients with impaired ability (81.2 years). Regarding sex, there were 18 men and 36 women in the group with unchanged ability to perform ADL group, whereas there were only three men and three women in the impaired ability group. The average waiting periods before surgery were 3.1 months in the unchanged ability group and 4.2 months in the impaired ability group.

Preoperative neurological disorders were detected in 28 patients in the group with unchanged ability to perform $\mathrm{ADL}$ and in three patients with impaired ability. The average number of comorbidities tended to be lower $(p=0.059)$ in the unchanged ability group ( 1.9 diseases per patient) than in those with impaired ability to perform ADL (three diseases per patient).

The average operative time was 237 minutes in the group with unchanged ability to perform ADL and 204 minutes in the impaired ability group. The average blood loss volumes were $421 \mathrm{~mL}$ in the unchanged ability group

Table 4. Radiological outcomes of patients in comparison between with DISH and without DISH

\begin{tabular}{lccc} 
Variable & $\begin{array}{c}\text { DISH }(+) \\
(\mathrm{n}=26)\end{array}$ & $\begin{array}{c}\text { DISH (-) } \\
(\mathrm{n}=37)\end{array}$ & $p$-value \\
\hline Previous vertebral fracture & 11 & 16 & 0.72 \\
\hline Three-column injury & 14 & 15 & 0.21 \\
\hline Middle column injury & 16 & 28 & 0.22 \\
\hline Corrective angle $\left(^{\circ}\right)$ & $17.2 \pm 10.5$ & $14.3 \pm 10.1$ & 0.27 \\
\hline Corrective loss $\left(^{\circ}\right)$ & $6.4 \pm 5.8$ & $11.1 \pm 9.0$ & $0.02^{*}$ \\
\hline Additional vertebral fracture & $2 / 24$ & $8 / 36$ & 0.19 \\
\hline Osteoporotic vertebral fracture fusion & $21 / 24$ & $20 / 36$ & $0.004^{*}$
\end{tabular}

Values are presented as number or mean \pm standard deviation. DISH, diffuse idiopathic skeletal hyperostosis. ${ }^{*} p<0.05$; statistically significant. 
and $343 \mathrm{~mL}$ in the impaired ability group. The average fusion levels were 4.5 in patients with unchanged ability to perform ADL and 3.8 for those with impaired ability. Perioperative complications occurred in 21 patients in the unchanged ability group and in four patients in the impaired ability group. Six patients in the unchanged ability group and two patients in the impaired ability group required revision surgery. These differences were not statistically significant (Table 5).

Regarding the preoperative VAS scores, the values were 7.5 in patients with unchanged ability to perform ADL and 7.1 in those with impaired ability, and this difference was not statistically significant.

Postoperatively, the VAS scores were significantly lower $(p=0.04)$ in those with unchanged ability to perform (2.2) than in those with impaired ability (3.1). The improvements in VAS scores were significantly higher $(p=0.03)$ in the unchanged ability group (68.9\%) than in the impaired ability group (51\%).

DISH-related complications occurred in 20 and three patients in the group with unchanged ability to perform $\mathrm{ADL}$ and the group with impaired ability to perform ADL, respectively. Previous vertebral fractures were detected in 24 and two patients in the unchanged ability group and the impaired ability group, respectively. Threecolumn injuries occurred in 26 and two patients in the unchanged ability group and the impaired ability group, respectively. Middle column injuries were detected in 34 and three patients in the unchanged ability group and the impaired ability group, respectively. The corrective angles

Table 5. Patient demographics and surgical outcomes in comparison between unchanged and impaired abilities to perform ADL

\begin{tabular}{|c|c|c|c|}
\hline Variable & Unchanged ADL group $(n=54)$ & Impaired ADL group ( $n=6)$ & $p$-value \\
\hline Age (yr) & $75.7 \pm 9.7$ & $81.2 \pm 3.7$ & $0.01 *$ \\
\hline Sex & & & 0.34 \\
\hline Male & 18 & 3 & \\
\hline Female & 36 & 3 & \\
\hline Waiting period (mo) & 3.1 & 4.2 & 0.19 \\
\hline Preoperative neurological disorders & 28 & 3 & 0.07 \\
\hline \multicolumn{4}{|l|}{ Frankel score } \\
\hline C & 16 & 3 & \\
\hline $\mathrm{D}$ & 12 & & \\
\hline Postoperative neurological disorders & 6 & 1 & 0.76 \\
\hline \multicolumn{4}{|l|}{ Frankel score } \\
\hline C & 1 & - & \\
\hline $\mathrm{D}$ & 5 & 1 & \\
\hline Comorbidity & $1.9 \pm 1.6$ & $3 \pm 1.7$ & 0.059 \\
\hline Surgical procedure & & & 0.23 \\
\hline PSF & 22 & 3 & \\
\hline$P S F+V P$ & 15 & - & \\
\hline $\mathrm{PSF}+\mathrm{PLIF}$ & 9 & 3 & \\
\hline APF & 8 & - & \\
\hline Operating time (min) & $237 \pm 97.6$ & $204 \pm 69.4$ & 0.32 \\
\hline Surgical blood loss (mL) & $421 \pm 331$ & $343 \pm 326$ & 0.59 \\
\hline Fusion level & $4.5 \pm 1.9$ & $3.8 \pm 1.9$ & 0.42 \\
\hline Perioperative complication & 21 & 4 & 0.19 \\
\hline Revision surgery & 6 & 2 & 0.17 \\
\hline
\end{tabular}

Values are presented as mean \pm standard deviation or number.

ADL, activity of daily living; PSF, posterior spinal fusion; VP, vertebroplasty; PLIF, posterior lumbar interbody fusion; APF, anterior and posterior fixation. $p<0.05$; statistically significant. 
were $16.4^{\circ}$ in the unchanged ability group and $13.5^{\circ}$ in the impaired ability group. The corrective loss was $9.1^{\circ}$ in the unchanged ability group and $12.5^{\circ}$ in the impaired ability group. Furthermore, additional vertebral fractures were detected in nine patients with unchanged ability to perform ADL and in one patient with impaired ability. Finally, OVF fusion was observed in 41 and five patients in the unchanged ability group and the impaired ability group, respectively. These differences were not statistically significant (Table 6).

\section{Discussion}

This study was conducted to identify the risk factors that have an impact on the ability to perform ADL after surgical treatment for OVF. Consequently, the following risk factors were identified to be associated with a reduced ability to perform ADL after conservative treatment for OVF: age $\geq 75$ years, female sex, middle column injury, previous vertebral fractures, and delayed union [2-4]. The mean age of our study patients in the group with impaired ability to perform ADL was significantly higher than that of patients with unchanged ability, but sex, middle column injury, previous vertebral fractures, and delayed union did not have much impact on their ability to perform ADL, possibly because surgical treatment might result in lower risk than conservative treatment for OVF.

In the present study, we also identified high postoperative VAS scores and low improvements in VAS scores as risk factors for an impaired ability to perform ADL after the surgical treatment for OVF. Although we observed that patients with DISH were likely to have complications such as thoracic and lumbar pain [5], both the preoperative and postoperative VAS scores and the improvements in VAS scores were similar between $\mathrm{D}+$ and $\mathrm{D}$ - patients.

One study reported that osteoporosis drugs are effective in relieving the pain of OVF [6]. Another study demonstrated that serotonin-norepinephrine reuptake inhibitors can effectively relieve postoperative residual pain [7]. These studies suggested that various aggressive drug therapies can improve postoperative pain, thereby leading to an improved ability to perform ADL after the surgical treatment for OVF.

Because patients with OVF are elderly and have various comorbidities, and also because surgical procedures vary depending on the hospital, there is a lack of substantial data in the literature [8]. We generally perform PSF, which is typically selected for the treatment of OVF. The results of the present study revealed that variations in surgical procedures (e.g., with or without anterior column reconstruction) had no significant impact on the ability to perform ADL. Kashii et al. [9] performed three different types of surgeries on patients with OVF and found no significant difference in the patients' ability to perform ADL. Although anterior column reconstruction is recommended for patients with OVF, to reduce the fixation range and the postoperative correction loss [10], it may not be necessary to improve the patient's ability to perform ADL.

Table 6. Clinical and radiological outcomes in comparison between unchanged and impaired abilities to perform ADL

\begin{tabular}{|c|c|c|c|}
\hline Variable & Unchanged ADL group ( $n=54)$ & Impaired ADL group (n=6) & $p$-value \\
\hline Preoperative VAS (cm) & $7.5 \pm 1.6$ & $7.1 \pm 2.3$ & 0.65 \\
\hline Postoperative VAS (cm) & $2.2 \pm 1.1$ & $3.1 \pm 1.8$ & $0.04^{*}$ \\
\hline Improvement rate of VAS (\%) & $68.9 \pm 19.0$ & $51 \pm 35.8$ & $0.03^{*}$ \\
\hline Complication with DISH & 20 & 3 & 0.91 \\
\hline Previous vertebral fracture & 24 & 2 & 0.44 \\
\hline 3-Column injury & 26 & 2 & 0.4 \\
\hline Middle column injury & 34 & 3 & 0.17 \\
\hline Corrective angle $\left({ }^{\circ}\right)$ & $16.4 \pm 10.6$ & $13.5 \pm 5.2$ & 0.14 \\
\hline Corrective loss $\left(^{\circ}\right)$ & $9.1 \pm 8.1$ & $12.5 \pm 9.2$ & 0.17 \\
\hline Additional vertebral fracture & 9 & 1 & 0.71 \\
\hline Osteoporotic vertebral fracture fusion & 41 & 5 & 0.79 \\
\hline
\end{tabular}

Values are presented as mean \pm standard deviation or number.

ADL, activity of daily living; VAS, Visual Analog Scale; DISH, diffuse idiopathic skeletal hyperostosis.

" $p<0.05$; statistically significant. 
In contrast, the rate of revision surgery for implant failure was higher in D+ patients; therefore, the fusion level for PSF in these may have been shorter.

The characteristics of vertebral fractures in patients complicated by DISH are likely to be accompanied by comorbidities such as hypertension, diabetes mellitus, and hyperlipidemia [11-14]. A multicenter study reported that at least $80 \%$ of the patients had a comorbidity, of whom approximately $50 \%$ had complications due to multiple comorbidities [15]. Our study also revealed significantly more comorbidities in the group with DISH complications, and that more comorbidities tended to cause an impaired ability to perform ADL. Therefore, patients with multiple comorbidities who underwent surgical treatment for OVF tended to have impaired abilities in performing ADL.

Surgical treatment of vertebral fractures in patients complicated by DISH is likely to result in frequent perioperative complications [16] and high mortality rates [17]. Our study also demonstrated a higher incidence of perioperative complications, revision surgery, and 1-year postoperative mortality in the group with DISH complications. Because DISH is an independent risk factor for mortality after vertebral fractures [16], the presence of DISH may indicate a high risk of developing complications at the time of surgery for OVF.

Older age has been reported as another risk factor for mortality after vertebral fractures [16]. The majority of research regarding spine fractures complicated with DISH (except for a study of three patients in their 90s [18]) involved patients in their 70 s or of a younger age $[11,16,19$ 23]. Therefore, there is a lack of substantial case reports of surgical treatment for older aged patients. In the present study, the majority of patients in the group with DISH complications were aged $\geq 80$ years and their postoperative abilities to perform ADL were as well-maintained as those of younger age patients without DISH. DISH may generate a need for bed rest for a long period of time due to the high mortality risk after conservative treatment for vertebral fractures [17] and the reduced physical function of the patient [14]. These results suggest that surgical treatment of elderly patients with OVF complicated by DISH can prevent disuse atrophy after injury. Furthermore, patients with DISH complications exhibited less postoperative correction loss and significantly higher rates of bone fusion postoperatively than those without DISH, possibly because of an increased ability of patients with DISH to form new bone [24]. However, as described, older age was identified as a postoperative risk factor for an impaired ability to perform ADL. Therefore, it would be beneficial to develop a future guideline such as a sliding scale for the surgical treatment of OVF.

This study had some limitations, such as the single-center retrospective design and a small sample size. As such, future investigations should include multicenter prospective analyses with larger sample sizes.

\section{Conclusions}

The majority of patients with OVF complicated by DISH are elderly men with multiple comorbidities and high postoperative mortality risks. However, patients with DISH complications have less correction loss and better bone fusion than those without DISH. In addition, preoperative and postoperative VAS scores, as well as improvements in VAS scores, appear to be similar between patients with and without DISH.

Older age, postoperative high VAS scores, and low postoperative improvements in VAS scores were associated with an impaired ability to perform ADL after the surgical treatment for OVF. The postoperative impaired ability to perform ADL is limitedly influenced by DISH. Therefore, we consider that surgical treatment of OVF combined with DISH is effective and appropriate for elderly patients.

\section{Conflict of Interest}

No potential conflict of interest relevant to this article was reported.

\section{Author Contributions}

Shinichi Kato: conception and design, analysis of data, drafting of the manuscript, critical revision; Nobuki Terada: administrative support; and Osamu Niwa: data acquisition.

\section{References}

1. Resnick D, Shaul SR, Robins JM. Diffuse idiopathic skeletal hyperostosis (DISH): Forestier's disease with extraspinal manifestations. Radiology 1975;115:51324.

2. Suzuki N, Ogikubo O, Hansson T. Previous vertebral 
compression fractures add to the deterioration of the disability and quality of life after an acute compression fracture. Eur Spine J 2010;19:567-74.

3. Matsumoto T, Hoshino M, Tsujio T, et al. Prognostic factors for reduction of activities of daily living following osteoporotic vertebral fractures. Spine (Phila Pa 1976) 2012;37:1115-21.

4. Takahashi S, Hoshino M, Takayama K, et al. Predicting delayed union in osteoporotic vertebral fractures with consecutive magnetic resonance imaging in the acute phase: a multicenter cohort study. Osteoporos Int 2016;27:3567-75.

5. Mader R, Lavi I. Diabetes mellitus and hypertension as risk factors for early diffuse idiopathic skeletal hyperostosis (DISH). Osteoarthritis Cartilage 2009;17:825-8.

6. Silverman SL, Minshall ME, Shen W, Harper KD, Xie S; Health-Related Quality of Life Subgroup of the Multiple Outcomes of Raloxifene Evaluation Study. The relationship of health-related quality of life to prevalent and incident vertebral fractures in postmenopausal women with osteoporosis: results from the Multiple Outcomes of Raloxifene Evaluation Study. Arthritis Rheum 2001;44:2611-9.

7. Koh IJ, Kim MS, Sohn S, Song KY, Choi NY, In Y. Duloxetine reduces pain and improves quality of recovery following total knee arthroplasty in centrally sensitized patients: a prospective, randomized controlled study. J Bone Joint Surg Am 2019;101:64-73.

8. Sheng X, Ren S. Surgical techniques for osteoporotic vertebral collapse with delayed neurological deficits: a systematic review. Int J Surg 2016;33 Pt A:42-8.

9. Kashii M, Yamazaki R, Yamashita T, et al. Surgical treatment for osteoporotic vertebral collapse with neurological deficits: retrospective comparative study of three procedures: anterior surgery versus posterior spinal shorting osteotomy versus posterior spinal fusion using vertebroplasty. Eur Spine J 2013;22:163342.

10. Katsumi K, Hirano T, Watanabe K, et al. Surgical treatment for osteoporotic thoracolumbar vertebral collapse using vertebroplasty with posterior spinal fusion: a prospective multicenter study. Int Orthop 2016;40:2309-215.

11. Caron T, Bransford R, Nguyen Q, Agel J, Chapman J, Bellabarba C. Spine fractures in patients with ankylosing spinal disorders. Spine (Phila Pa 1976)
2010;35:E458-64.

12. Kiss C, Szilagyi M, Paksy A, Poor G. Risk factors for diffuse idiopathic skeletal hyperostosis: a case-control study. Rheumatology (Oxford) 2002;41:27-30.

13. Miyazawa N, Akiyama I. Diffuse idiopathic skeletal hyperostosis associated with risk factors for stroke: a case-control study. Spine (Phila Pa 1976) 2006;31:E225-29.

14. Katzman WB, Huang MH, Kritz-Silverstein D, Barrett-Connor E, Kado DM. Diffuse idiopathic skeletal hyperostosis (DISH) and impaired physical function: the Rancho Bernardo study. J Am Geriatr Soc 2017;65:1476-81.

15. Okada E, Tsuji T, Shimizu K, et al. CT-based morphological analysis of spinal fractures in patients with diffuse idiopathic skeletal hyperostosis. J Orthop Sci 2017;22:3-9.

16. Westerveld LA, van Bemmel JC, Dhert WJ, Oner FC, Verlaan JJ. Clinical outcome after traumatic spinal fractures in patients with ankylosing spinal disorders compared with control patients. Spine J 2014;14:72940.

17. Westerveld LA, Verlaan JJ, Oner FC. Spinal fractures in patients with ankylosing spinal disorders: a systematic review of the literature on treatment, neurological status and complications. Eur Spine J 2009;18:145-56.

18. Tanishima S, Takeda C, Hamamoto Y, Kondo Y, Nagashima H. Prompt surgical management for spinal fracture in the elderly aged over 90 years with diffuse idiopathic skeletal hyperostosis to extend their healthy lifespan. Eur J Orthop Surg Traumatol 2012;22 Suppl 1:29-34.

19. Whang PG, Goldberg G, Lawrence JP, et al. The management of spinal injuries in patients with ankylosing spondylitis or diffuse idiopathic skeletal hyperostosis: a comparison of treatment methods and clinical outcomes. J Spinal Disord Tech 2009;22:77-85.

20. Paley D, Schwartz M, Cooper P, Harris WR, Levine AM. Fractures of the spine in diffuse idiopathic skeletal hyperostosis. Clin Orthop Relat Res 1991:22-32.

21. Le Hir PX, Sautet A, Le Gars L, et al. Hyperextension vertebral body fractures in diffuse idiopathic skeletal hyperostosis: a cause of intravertebral fluidlike collections on MR imaging. AJR Am J Roentgenol 1999;173:1679-83.

22. Bransford RJ, Koller H, Caron T, et al. Cervical spine 
trauma in diffuse idiopathic skeletal hyperostosis: injury characteristics and outcome with surgical treatment. Spine (Phila Pa 1976) 2012;37:1923-32.

23. Meyer PR Jr. Diffuse idiopathic skeletal hyperostosis in the cervical spine. Clin Orthop Relat Res 1999:4957.
24. Baraliakos X, Listing J, Buschmann J, von der Recke A, Braun J. A comparison of new bone formation in patients with ankylosing spondylitis and patients with diffuse idiopathic skeletal hyperostosis: a retrospective cohort study over six years. Arthritis Rheum 2012;64:1127-33. 